\title{
Teaching a Neural Network the Hard Way
}

\section{A neural network can be made to produce more reliable predictions of} nonlinear systems if it is created with conservation laws built in.

\author{
By Marric Stephens
}

$\Lambda$ baby learns by observation how objects move. But without accounting for conservation of momentum, its developed understanding is just an educated guess. Similarly, an artificial neural network (ANN) learns from empirical data how a particular system works, but without explicitly considering the conservation laws that govern that system, it risks making unreliable predictions. To address this limitation, Tom Beucler at the University of California, Irvine, and colleagues have devised a way to hardwire an ANN with such laws. They demonstrated the technique using an atmospheric model for the climate, but they say that their method can be applied to models of any physical system [1].

An ANN is an algorithm-based tool for turning a set of inputs into a set of outputs. The development or "training" process for an ANN involves incrementally reconfiguring its underlying algorithm with data from observations until the outputs accurately match reality. During this process, a conventional ANN can be discouraged from making physically impossible predictions by imposing "soft constraints" on its

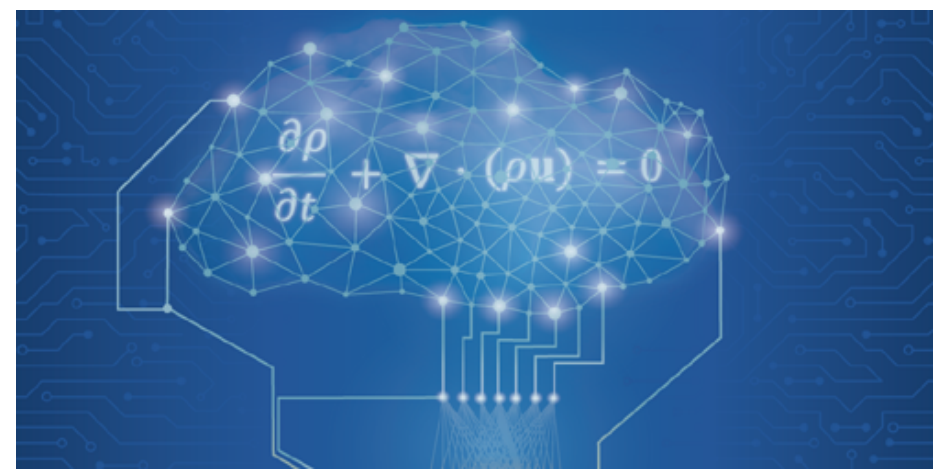

Credit: R. Gauthier-Butterfield/UC Irvine outputs-rewarding outcomes that better conform to physical laws.

Beucler and colleagues used an ANN to simulate the climatic effects of atmospheric convection. Such models are reliable only if they respect strict conservation of mass and energy. Instead of imposing these laws using soft constraints, the team embedded them in the architecture of the ANN as "hard constraints." Likened to an infant's learning process, the method is equivalent to making physics-defying predictions literally unthinkable.

The addition of these hard constraints makes it more difficult for the ANN to reach its optimal output. The researchers say that this price is worth paying though, as conservation laws are critical to climate models.

Marric Stephens is a Corresponding Editor for Physics based in Bristol, UK.

\section{REFERENCES}

1. T. Beucler et al., "Enforcing analytic constraints in neural networks emulating physical systems," Phys. Rev. Lett. 126, 098302 (2021). 